\title{
Optimisation and Statistical Analysis of 21.4 Gb/s RZ-DPSK WDM Non-Slope Matched Transmission
}

\author{
Terence Broderick*a , Sonia A. Boscolo ${ }^{\mathrm{a}}$, Wai Wong ${ }^{\mathrm{b}}$ \\ ${ }^{a}$ Photonics Research Group, Aston Univ., Birmingham, UK B4 7ET; \\ ${ }^{\mathrm{b}}$ Azea Networks Ltd, Bates House, Harold Wood, Romford RM3 0SD
}

\begin{abstract}
Considering a numerical example, we analyse the performance of Return-to-Zero (RZ) Differential Phase Shift Keyed (DPSK) transmission when deployed in a large scale transmission system. It is shown that at high distances, RZ-DPSK performs well whilst being limited by nonlinear effects. We also show that when nonlinear effects become dominant, we can still estimate channel statistics to reasonable accuracy.
\end{abstract}

Keywords: Differential phase-shift keying, statistical analysis, bit-error rate

\section{INTRODUCTION}

Upgrades of installed submarine systems designed for $\mathrm{Nx} 2.5 \mathrm{~Gb} / \mathrm{s}$ or $\mathrm{Nx} 10 \mathrm{~Gb} / \mathrm{s}$ bit-rate and on-off keying (OOK) modulation have recently attracted considerable attention [1-3]. These systems, which have been installed in large numbers over the last decade, usually use a mix of negative dispersion, non-zero dispersion shifted fibre (NZ-DSF) and standard single mode fibre (SSMF). It has been recently shown [1-5], that the adaptation of these links to the differential phase-shift keying (DPSK) modulation format, shown to exhibit $3 \mathrm{~dB}$ receiver sensitivity improvement relative to OOK, is not straightforward. It has been shown however, that $50 \mathrm{GHz}$ spaced $20 \mathrm{~Gb} / \mathrm{s}$ return-to-zero (RZ) DPSK i.e high spectral efficiency $(0.4 \mathrm{bits} / \mathrm{s} / \mathrm{Hz})$ is a promising candidate for upgrades of installed submarine systems [6]. The application of DPSK to submarine systems has already been demonstrated in both the $10 \mathrm{~Gb} / \mathrm{s}$ [7], 20Gb/s $[2,4]$ and $40 \mathrm{~Gb} / \mathrm{s}$ [8], all being at the same spectral efficiency. It has also been shown [2,4] that varying the pulse width allows the realisation of quite different transmission regimes.

The major goal of this paper is to theoretically assess the bit-error rate (BER) performance of a typical non-slope matched transoceanic WDM submarine transmission link using 50-GHz spaced RZ-DPSK at a bit rate of $21.4 \mathrm{Gbit} / \mathrm{s}$ $(\sim 0.4 \mathrm{bit} /(\mathrm{s} \mathrm{Hz})$ spectral efficiency) with different duty cycles of the signal pulses. Note that a variety of new transmission regimes similar to that in high-bit-rate systems can be applied even in existing systems operating at lower bit rate. For instance, as it was pointed out in [10], a pseudo-linear, bit-overlapping transmission regime that is typically attributed to $40 \mathrm{Gbit} / \mathrm{s}$ systems can be advantageously applied at $10 \mathrm{Gbit} / \mathrm{s}$ rates using short carrier pulses and RZ-DPSK format. We would like to stress that by varying the pulse duty cycle at $21.4 \mathrm{Gbit} / \mathrm{s}$ channel rate it is possible to realise quite different transmission regimes, ranging from a pseudo-linear regime to a dispersion-managed soliton-like one. We also discuss the reliability of different available Q-factor approaches to the BER estimation for the RZ-DPSK format $[11,12]$, and investigate their accuracy with respect to the different duty cycles by comparing their predictions to the results of direct error counting. Firstly, we optimize the pre/post compensation map. This allows the study of the operational regimes allowed for the three duty cycles considered $33 \%, 50 \%$ and $67 \%$. We then show the evolution of the BER for increased distances, demonstrating the validity of the three BER estimation methods explored in [13]. Following an analysis of the BER estimation, we consider the case when the conventional Gaussian estimate based on electrical Q-factor fails. This leads to the consideration of new statistical estimation procedures for BER when the transmission is dominated by fibre nonlinearity such as intra-channel four-wave mixing (IFWM) and intra-channel crossphase modulation (IXPM) as in the pertinent quasi-linear transmission regime [14].

*broderit@aston.ac.uk

Optical Technologies for Telecommunications 2008, edited by Vladimir A. Andreev,

Vladimir A. Burdin, Oleg G. Morozov, Albert H. Sultanov, Proc. of SPIE Vol. 7374,

$737402 \cdot$ @ 2009 SPIE · CCC code: 0277-786X/09/\$18 - doi: 10.1117/12.828991

Proc. of SPIE Vol. 7374 737402-1 


\section{SYSTEM DESCRIPTION AND NUMERICAL MODELLING}

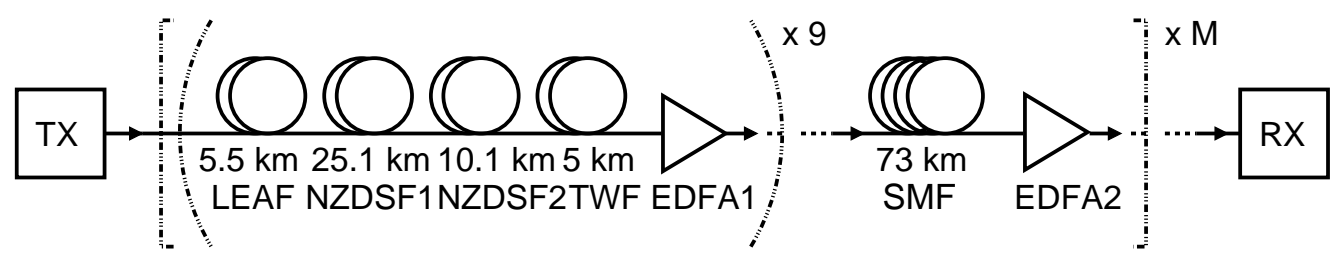

Fig.1. Setup of the transmission link.

The transmission link used in our analysis is depicted in Figure 1. We choose to emulate a non-slope matched submarine type link that is implemented in our experiments as a re-circulating fibre twin-loop. This offers the advantage of high flexibility and allows replication of the dispersion and optical signal-to-noise ratio maps of a typical transoceanic submarine link. The unit cell comprises nine NZ-DSF sections with negative dispersion. Each of them has a length of $45.7 \mathrm{~km}$ and is composed of a mix of large effective core area fibre (LEAF), standard NZ-DSF and reduced slope fibre (TrueWave fibre), followed by a SSMF section of length $73.2 \mathrm{~km}$. The residual path average dispersion of the line at $1550 \mathrm{~nm}$ is $-0.025 \mathrm{ps} / \mathrm{nm}$.

Table 1. Fibre parameters.

\begin{tabular}{|l|c|c|c|c|c|}
\hline Fibre & LEAF & NZDSF1 & NZDSF2 & TWF & SMF \\
\hline $\mathbf{D}[\mathbf{p s} / \mathbf{n m}]$ & -2.8 & -3.07 & -2.91 & -3.26 & 16.79 \\
\hline S[ps/nm2km] & 0.11 & 0.09 & 0.09 & 0.04 & 0.06 \\
\hline $\boldsymbol{\alpha}[\mathbf{d B} / \mathbf{k m}]$ & 0.21 & 0.20 & 0.21 & 0.20 & 0.19 \\
\hline$\gamma[\mathbf{1} / \mathbf{W} \mathbf{k m}]$ & 1.27 & 1.88 & 1.85 & 1.99 & 1.19 \\
\hline Length[km] & 5.5 & 25.1 & 10.1 & 5.0 & 73.2 \\
\hline
\end{tabular}

Erbium-doped fibre amplifiers (EDFAs) with a noise figure of $4.5 \mathrm{~dB}$ are employed to compensate for the losses in both the fibres and the splicing associated with the fibre joins. The unit cell of the system is iterated M times. The transmitter is modelled as the concatenation of a distributed feedback laser, a dual-drive Mach-Zehnder modulator-based pulse carver with an extinction ratio of $30 \mathrm{~dB}$, and an ideal phase modulator. Three biasing configurations of the pulse carver are used to create RZ pulses with duty cycles $33 \%, 50 \%$ and $67 \%$ at a $21.4 \mathrm{~Gb} / \mathrm{s}$ bit-rate in accordance with the power transfer function given below

$$
T(t)=\left|\frac{u_{\text {out }}}{u_{\text {in }}}\right|^{2}=\frac{1}{4}\left\{1+\gamma^{2}+2 \gamma \cos \left[\frac{\left.\pi\left(2 V_{1} \cos (2 \pi \varpi t+\theta)\right)-V_{b}\right)}{V_{\pi}}\right]\right\},
$$

where the $u$ fields correspond to the input and output fields respectively, $\mathrm{V}_{\pi}$ is the voltage required to produce a $\pi$ phase shift, $\mathrm{V}_{\mathrm{b}}$ is a constant bias voltage and we define $\gamma$, the depth of the modulation, as below

$$
\gamma=\frac{\sqrt{\varepsilon}-1}{\sqrt{\varepsilon}+1}
$$

Where $\varepsilon$ is the extinction ratio.

We consider 5 equally spaced $(0.4 \mathrm{~nm})$ channels centred symmetrically upon $1550 \mathrm{~nm}$. The channels are de-multiplexed using a second-order Gaussian optical band-pass filter, detected using a balanced Mach-Zehnder delay interferometer, and then filtered electrically by a third-order Bessel filter with a cut-off frequency equal to the bit-rate. 


\section{PRE/POST-COMPENSATION OPTIMISATION}

First, we optimise the pre- and post-compensation dispersions of the system and the launch signal power for the different input pulse duty cycles used in this work. We use the electrical Q-factor as an indicator of the system performance. The Q-factor is calculated at the end of the unit cell of the link, and averaged over a number of 1024-bit pattern runs. The 3$\mathrm{dB}$ bandwidth of the de-multiplexer is optimized for each duty cycle through back-to-back simulations. Figure 2 shows contour-plots of the $\mathrm{Q}^{2}$-factor of the weakest channel at the output of the transmission link in the plane pre-compensation dispersion $\left(D_{\text {pre }}\right.$, given at $\left.1550 \mathrm{~nm}\right)$ - launch average power per channel. Here, the post-compensation dispersion, $D_{\text {post}}$, is selected such that $D_{\text {post }}=-D_{\text {pre }}-D_{\text {ave }} L+\delta D$, i.e. fine tuning of the pre/post compensation ratio is performed by addition of a dispersion amount $\delta \mathrm{D}$ in addition to the product $\mathrm{D}_{\mathrm{ave}} \mathrm{L}$. The results are shown at the optimum $\delta \mathrm{D}$. $\mathrm{D}_{\text {ave }}$ is the net-link dispersion $(-0.025 \mathrm{ps} / \mathrm{nm}$ at $1550 \mathrm{~nm})$ and $\mathrm{L}$ is the length of one span $(484 \mathrm{~km})$.

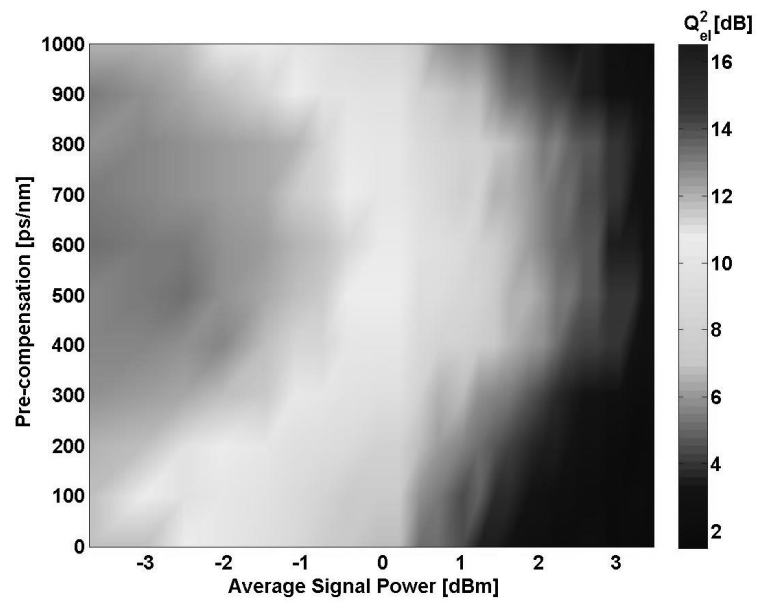

a)

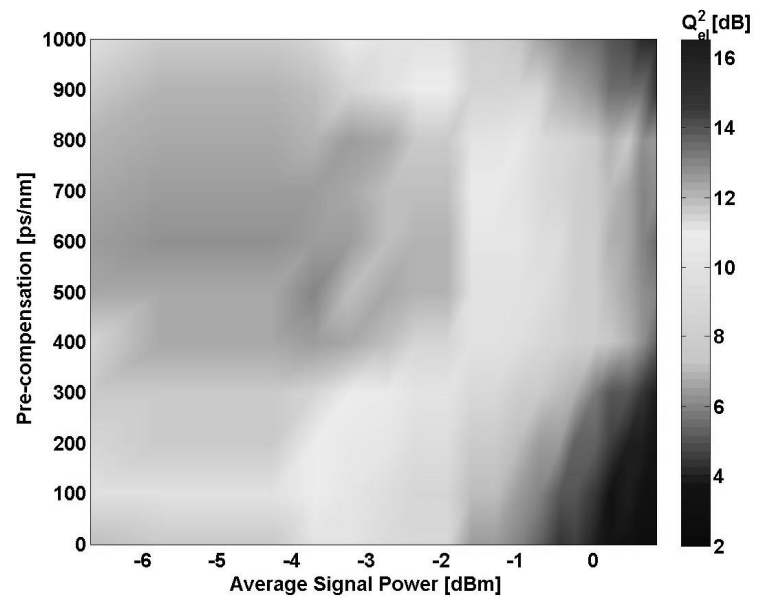

b)

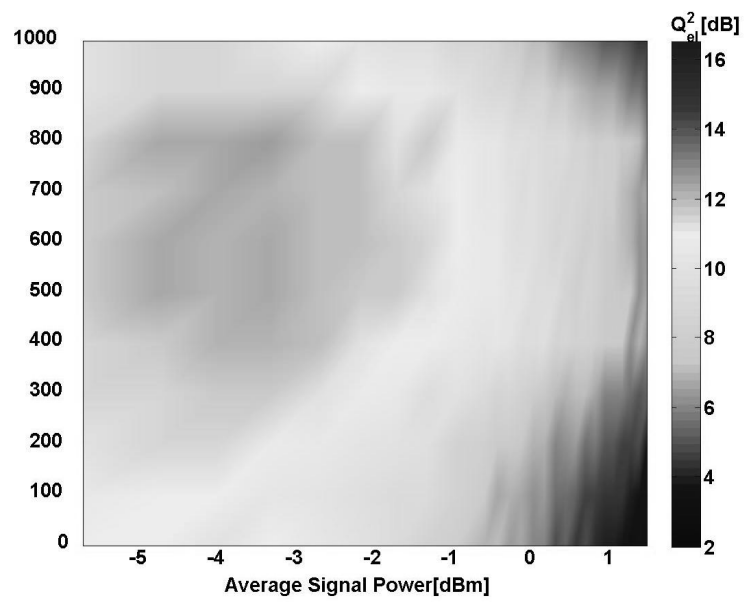

c)

Fig. 2. Weakest channel performance versus average signal power (Pave) and pre-compensation (Dpre) for a) $33 \%$ duty cycle, b) $50 \%$ duty cycle and c) $67 \%$ duty cycle.

It can be seen that the optimum pre-compensation and average power vary from approximately $(600 \mathrm{ps} / \mathrm{nm},-3 \mathrm{dBm})$ for the $33 \%$ duty cycle, to approximately $(700 \mathrm{ps},-3.7 \mathrm{dBm})$ for the $50 \%$ duty cycle, and to $(700 \mathrm{ps} / \mathrm{nm},-3.5 \mathrm{dBm})$ for the $67 \%$ duty cycle, while there are sufficiently large power margins within the dispersion range $400 \mathrm{ps} / \mathrm{nm}$ to $900 \mathrm{ps} / \mathrm{nm}$ for all duty cycles. It is also seen that narrower pulses than the conventional $50 \%$ duty-cycle pulses offer superior performance in terms of higher Q-values, as it is observed in the case of a single-channel transmission. It is seen that generally, $33 \%$ duty cycle pulses perform at a superior level to the two wider duty cycles. 


\section{BIT-ERROR RATE}

In our modelling, we compute the signal BER by direct counting of errors. We also use BER estimates in terms of the signal Q-factor, which is closely related to the error probability and is a widely used tool to estimate the performance of optical transmission systems because it is relatively easy to evaluate, thus preventing the need for time-consuming direct counting. We use here the standard Q-factor of the received electrical signal $\left(\mathrm{Q}_{\mathrm{el}}\right)$ [11], and the Q-factors defined for the optical (differential) phase $\left(\mathrm{Q}_{\varphi}\right)$ and the field amplitude before demodulation $\left(\mathrm{Q}_{\mathrm{A}}\right)$ of the signal, which were introduced in [12] to estimate the BER in a DPSK channel in the nonlinear and linear regimes, respectively. The BER is estimated from $\mathrm{Q}_{\mathrm{el}}, \mathrm{Q}_{\varphi}$ and $\mathrm{Q}_{\mathrm{A}}$ using the expressions: $\operatorname{BER}\left(\mathrm{Q}_{\mathrm{el}}\right)=(1 / 2) \operatorname{erfc}\left(\mathrm{Q}_{\mathrm{el}} / \sqrt{2}\right), \operatorname{BER}\left(\mathrm{Q}_{\varphi}\right)=\operatorname{erfc}\left(\mathrm{Q}_{\varphi /} \sqrt{2}\right)$ and $\mathrm{BER}\left(\mathrm{Q}_{\mathrm{A}}\right)=$ $(1 / 2) \exp \left(-Q^{2}{ }_{A} / 2\right)[12,13]$, which are calculated using the Gaussian approximation of the intensity noise, the field amplitude noise and the noise at the centre of each rail of the differential phase eye diagram, respectively.

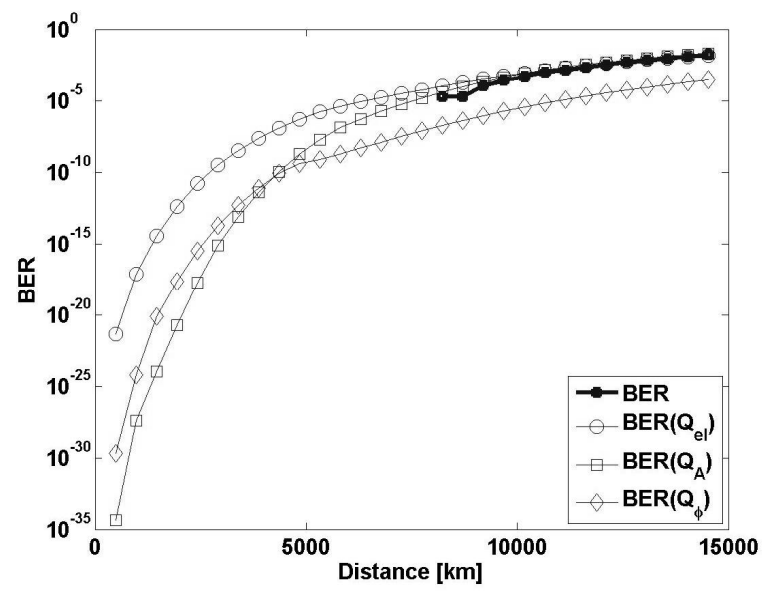

a) $33 \%$ duty cycle

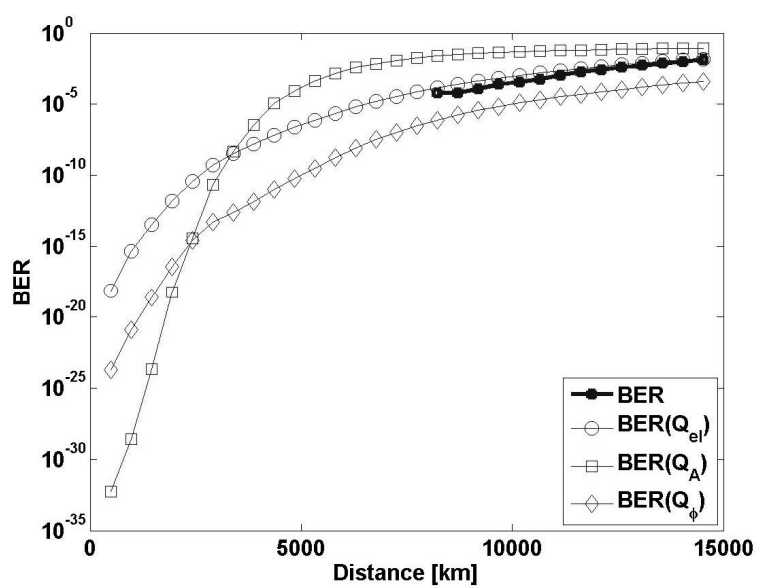

b) $50 \%$ duty cycle

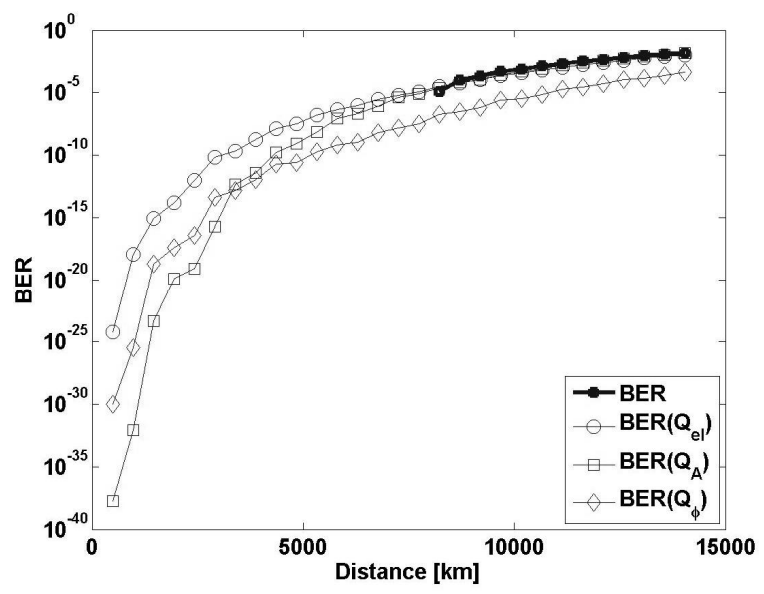

c) $67 \%$ duty cycle

Fig. 3. Theoretical BERs and directly computed BER versus distance for $50 \mathrm{GHz}$-spaced $21.4 \mathrm{Gbit} / \mathrm{s}$ WDM RZ-DPSK transmission.

Comparison of the accuracy of these Q-factor models versus the directly computed BER is given in Fig. 3, which shows the evolution of the theoretical BERs and the actual BER over the transmission distance at the optimal launch average 
power given by the results in Fig. 2. It can be seen that for all duty cycles $\mathrm{Q}_{\mathrm{A}}$ and $\mathrm{Q}_{\mathrm{el}}$ compete to one another at yielding the most reliable estimate of the actual BER, and the predictions based on these Q-factor models are more accurate at large distances. On the other hand, $\mathrm{Q}_{\varphi}$ significantly underestimates the actual BER. This is an indication that the transmission regime is quasi-linear [12]. The fact that the conventional electrical Q-factor is a fairly performance indicator would suggest that electrical current statistics in such channels are largely Gaussian - these assumptions have been subject to recent investigation [15]. It is also seen clearly from Fig. 3 that the $33 \%$ duty cycle pulses achieve the highest BER margin, which confirms the conclusion drawn from Fig. 2. Indeed, supposing an extension of the directly computed BER curves to the region of short distances with either $B E R\left(Q_{A}\right)$ or $B E R\left(Q_{e l}\right)$ as a reference curve, a $B E R$ of $10^{-9}$ would relate to a transmission distance of approximately $4500 \mathrm{~km}$ for the $33 \%$ duty cycle, $5000 \mathrm{~km}$ for the $50 \%$ duty cycle, and $5000 \mathrm{~km}$ for the widest duty cycle. In any case, the results for the three duty cycles studied show that errorfree $21.4 \mathrm{Gbit} / \mathrm{s}$ RZ-DPSK transmission at $50 \mathrm{GHz}$ channel separation is feasible beyond the design length of typical installed submarine links with no need for in-band slope compensation of chromatic dispersion, which confirms the experimental observations and theoretical predictions of previous works [5-7].

\section{STATISTICAL ANALYSIS}

We now consider the case when the Gaussian statistics do not apply. The efficient growth in capacity of optical communications systems is achieved by an increase in the channel bit-rate, assuming the use of shorter carrier pulses. Due to such narrow pulses, high capacity transmission is strongly influenced by the fibre dispersion. This results in large temporal broadening of the carrier pulses. Signal transmission using such short pulses is known as the quasi-linear regime. This regime is dominated by two sources of nonlinearity: IFWM and IXPM [14]. For suitably optimised configurations, RZ-DPSK can suppress this nonlinearity, providing superior performance over RZ-OOK [16]. However, when transmission distances and pulse powers increase such effects influence transmission to such an extent that channel statistics are altered. This is due to the raised interaction between pulses within a channel.

We study the statistics, and subsequently, the BER of the centre channel for the $50 \%$ duty cycle pulses considered in the previous sections. We consider the average signal power of $-0.8 \mathrm{dBm}$ at the optimal pre-compensation dispersion. From Fig. 2, we see that such a configuration provides less than optimal transmission. The raised level of power induces increased fibre nonlinearity [17], which is demonstrated in the eye diagrams given in Fig. 4 through the abundance of amplitude noise (due to IFWM) and timing jitter (due to IXPM).

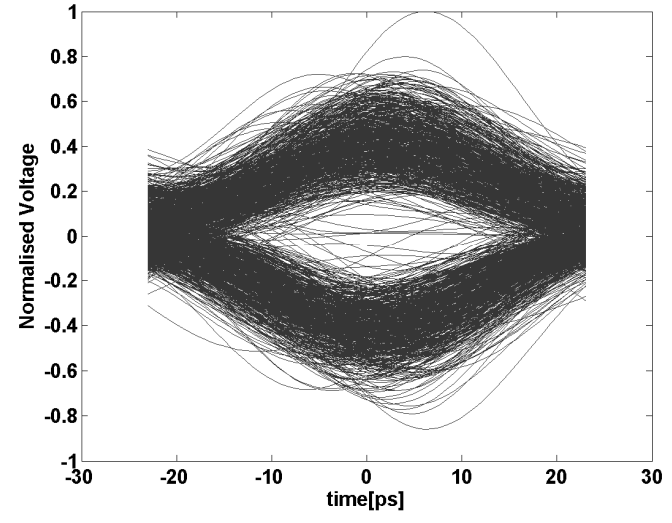

a)

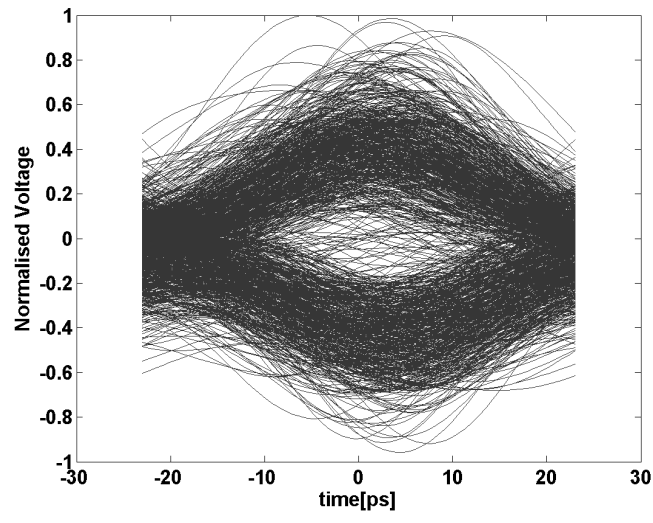

b)

Fig. 4. 50\% duty cycle pulses eye diagrams at $P_{a v e}=-0.8 \mathrm{dBm}$, at a) $7500 \mathrm{~km}$ and b) $10000 \mathrm{~km}$ transmission distance.

Interactions due to fibre nonlinearity can be measured using the kurtosis of the received current [18]. Kurtosis (K) is defined as the fourth standardized moment of the data. For Gaussian distributed data, $\mathrm{K}=3$, providing a simple reference point from which we can study the statistics of the channel. Figure 5 shows the evolution of the kurtosis of the received current for logical ones and zeros over the transmission distance. Results relative to the optimal average signal power of - 
$3.7 \mathrm{dBm}$ (see Fig. 2) are also reported for comparison. The increase of the kurtosis with distance at Pave $=-3.7 \mathrm{dBm}$ is due to the accumulation of nonlinear effects during transmission. Substantial deviation of the kurtosis of the "ones" distribution from the Gaussian value is also evident at $\mathrm{P}_{\text {ave }}=-0.8 \mathrm{dBm}$ at any of the considered distances. This is expected due to the increase in fibre nonlinearity commensurate with an increase in signal power.

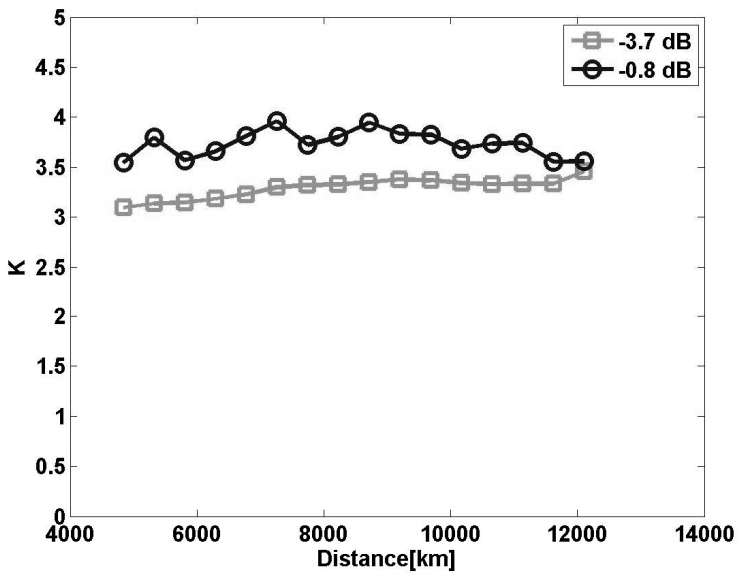

a)

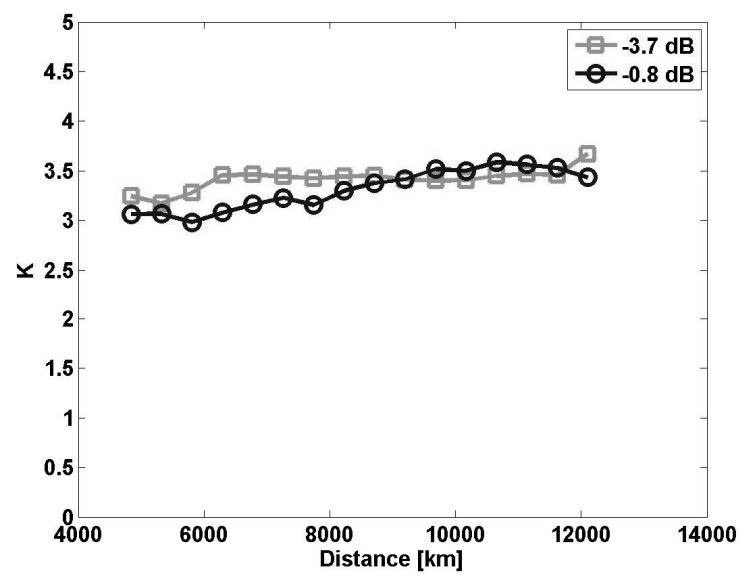

b)

Fig. 5. Kurtosis versus transmission distance for a) logical ones and b)logical zeros at $P_{\text {ave }}=-0.8 \mathrm{dBm}$ and $\mathrm{P}_{\text {ave }}=-3.7 \mathrm{dBm}$.

We now study the underlying received current histograms and subsequent fitting of the Gaussian distribution by comparison to the Gamma distribution. The Gamma distribution is an exponential distribution with probability density function given by

$$
f(I ; k, \theta)=\frac{I^{k-1} e^{-\frac{I}{\theta}}}{\Gamma(k) \theta^{k}}
$$

The distribution in (3) is characterised by two parameters, a shape parameter $\mathrm{k}$ and a scale parameter $\theta$. The underlying random variable can take a Gaussian shape when its shape parameter when $\mathrm{k}$ is large. It also possesses the property that kurtosis and skewness are shape dependent, allowing the modeling of more dynamic random phenomena, such as data transmission channels under the influence of IFWM and IXPM.

Analysis of the received current histograms (Fig. 6), suggests that the statistics corresponding to the eye diagrams in Fig. 4 and the kurtosis in Fig. 5 demonstrate behaviour comparable with that of the Gamma distribution. Non-Gaussian tail behaviour indicates increased levels of interaction between pulses, which causes the assumption of Gaussian statistics to be invalidated. Next, we transmit several patterns of length 10240 bits at average pulse powers of $-0.8 \mathrm{dBm}$ and $1.6 \mathrm{dBm}$ (less than optimal), and we compare the estimate for BER based on the Gamma distribution given in (4) as taken from [18], with the results of direct detection simulations and BER calculated from the electrical Q-factor. The parameters $\mathrm{k}$ and $\theta$ can be estimated directly from electrical current statistics as given below $(5,6)$;

$$
\begin{gathered}
B E R=\frac{1}{2}\left(1-\frac{\gamma\left(k_{1}, \frac{I_{d}}{\theta_{1}}\right)}{\Gamma\left(k_{1}\right)}-\frac{\gamma\left(k_{0}, \frac{I_{d}}{\theta_{0}}\right)}{\Gamma\left(k_{0}\right)}\right), \\
k_{1,0}=\frac{\mu_{1,0}^{2}}{\sigma_{1,0}^{2}},
\end{gathered}
$$




$$
\theta_{1,0}=\frac{\sigma_{1,0}^{2}}{\mu_{1,0}} .
$$

Where $\mu$ is the mean of the electrical current data and $\sigma$ is the standard deviation of the electrical current data.

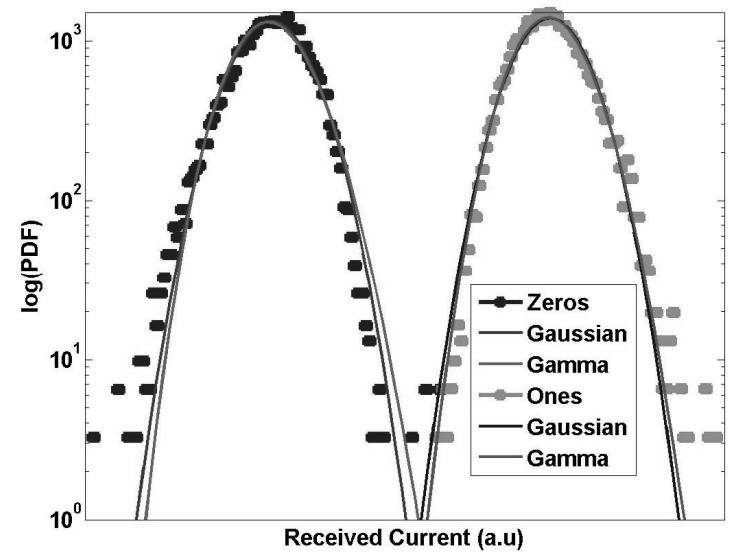

a)

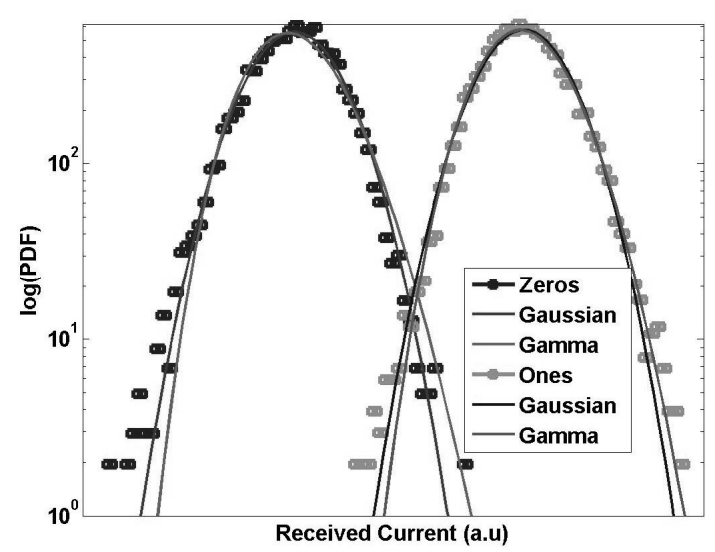

b)

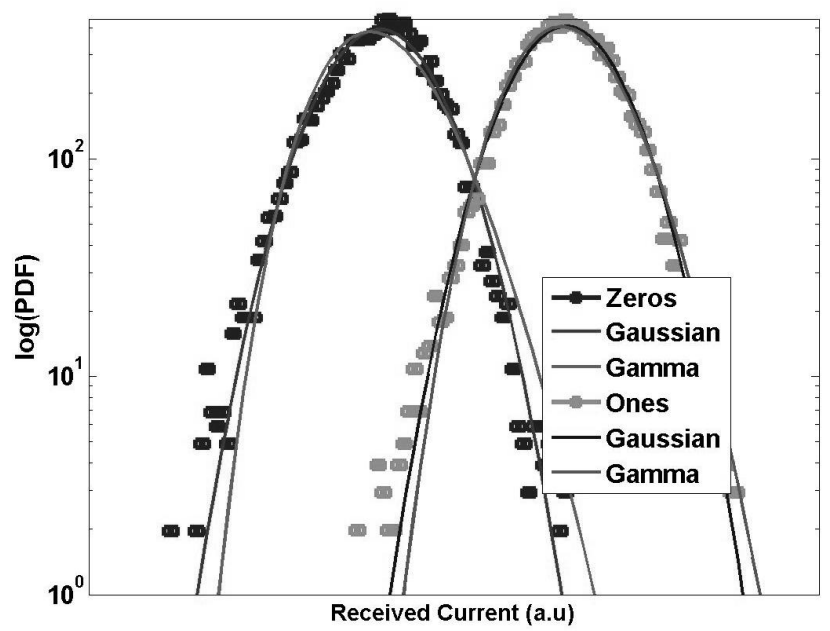

c)

Fig. 6. Received current histograms at $7500 \mathrm{~km}$ for a) $P_{a v e}=-3.7 \mathrm{dBm}$, b) $P_{a v e}=-1.6 \mathrm{dBm}$ and c) $P_{\text {ave }}=-0.8 \mathrm{dBm}$.

Given in Fig. 7, we can see that for these pulse powers, the subsequent increase in fibre nonlinearity gives the consequence of significant under-estimation of BER using the electrical Q-factor method, whereas estimation based on the more flexible Gamma distributed statistics provides accuracy in the estimation, thus offering an alternative for transmission dominated by fibre nonlinearity. 


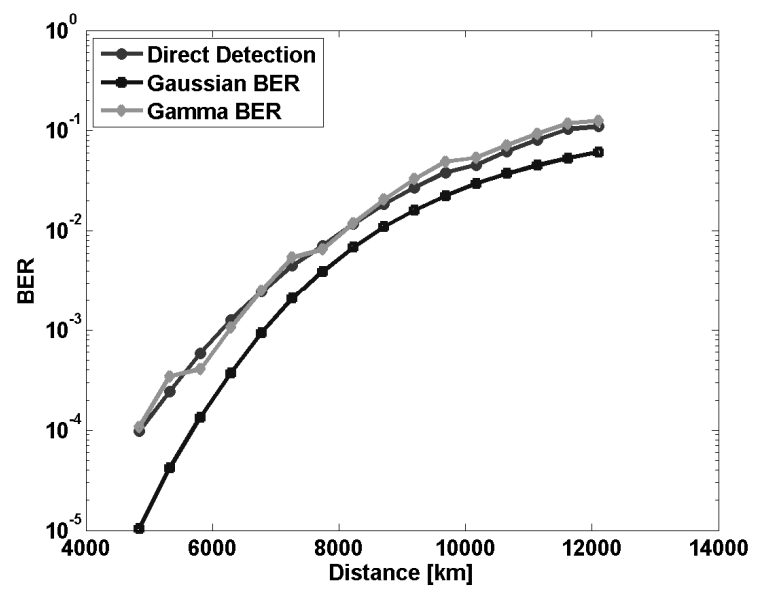

a)

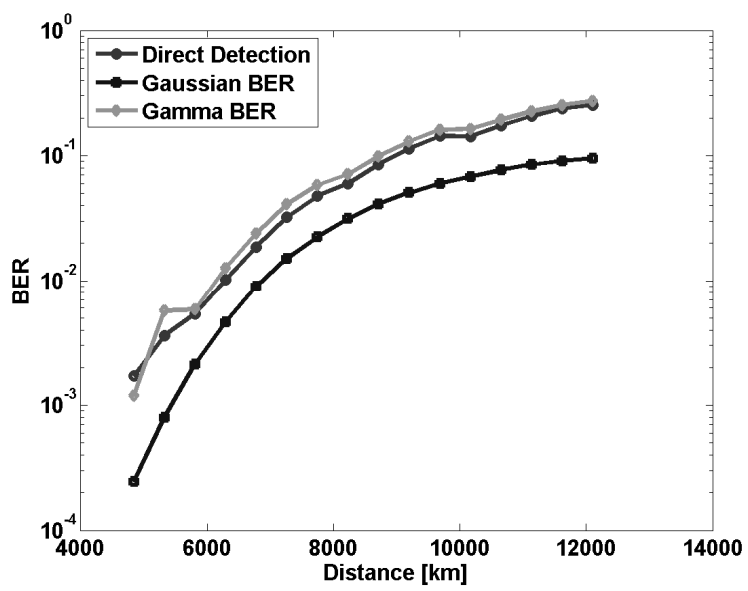

b)

Fig. 7. Bit-error rate evolution over transmission distance for a) $P_{a v e}=-0.8 \mathrm{dBm}$ and b) $P_{a v e}=1.6 \mathrm{dBm}$.

\section{CONCLUSION}

Firstly, we studied the performance of non-slope matched $21.4 \mathrm{~Gb} / \mathrm{s}$ WDM submarine transmission through optimization of the pre/post-compensation parallel to the pulse average power. We saw that for wider duty cycles, increased flexibility with regard to pulse average power was obtained at the expense of performance. Using the optimised configurations, we then studied the evolution of the BER using direct error counting. It was shown that without any in-line compensation of chromatic dispersion, optical regeneration at the receiver or forward-error correction, a BER of $10^{-9}$ was obtainable at transmission distances of at least $4500 \mathrm{~km}$. By comparison to the four traditional methods of Q-factor based BER estimation, we saw that estimation based on the electrical Q-factor was the most accurate, suggesting Gaussian electrical statistics. Finally, we studied statistical behaviour of the transmission at average signal powers higher than optimal average power. We compared the estimation of BER using electrical Q-factor with estimation based on the Gamma distribution. It was shown that for such high powers, where the transmission is dominated by fibre nonlinearity, BER estimation based on the Gamma distribution is valid. This signals a significant step forward in the analysis of such transmission.

\section{REFERENCES}

[1] J. -X. Cai, D. G. Foursa, L. Liu, C. R. Davidson, Y. Cai, W. W. Patterson, A. J. Lucero, B. Bakhshi, G. Mohs, P. C. Corbett, V. Gupta, W. Anderson, M. Vaa, G. Domagala, M. Mazurczyk, H. Li, S. Jiang, M. Nissov, A. N. Pilipetskii, and N. S. Bergano, "RZ-DPSK field trial over $13100 \mathrm{~km}$ of installed non-slope-matched submarine fibers," J. Lightwave Technol., vol. 23, pp. 95-103, 2005.

[2] J. -X. Cai, C. R. Davidson, M. Nissov, H. Li, W. Anderson, Y. Cai, L. Liu, A. N. Pilipetskii, D. G. Foursa, W. W. Patterson, P. C. Corbett, A. J. Lucero, and N. S. Bergano, "Transmission of $40 \mathrm{~Gb} / \mathrm{s}$ WDM signals over 6,250 km of conventional NZ-DSF with > 4 dB FEC margin," in Tech. Dig. of Optical Fiber Communication Conference (OFC 2005) Anaheim, CA, Mar. 2005, paper PDP 26.

[3] J. -X. Cai, M. Nissov, H. Li, C. R. Davidson, W. Anderson, L. Liu, D. G. Foursa, A. N. Pilipetskii, and N. S. Bergano, "Experimental comparison of $40 \mathrm{~Gb} / \mathrm{s}$ RZ-, CSRZ-, and NRZ-DPSK modulation formats over non slopematched fibres," in Proc. of 31st European Conference on Optical Communication (ECOC 2005), Glasgow, United Kingdom, Sept. 2005, paper Th1.2.2.

[4] J. -X. Cai, M. Nissov, W. Anderson, M. Vaa, C. R. Davidson, D. G. Foursa, L. Liu, Y. Cai, A. J. Lucero, W. W. Patterson, P. C. Corbett, A. N. Pilipetskii, and N. S. Bergano, "Long-haul 40 Gb/s RZ-DPSK transmission with long repeater spacing," in Tech. Dig. of Optical Fiber Communication Conference (OFC 2006), Anaheim, CA, Mar. 2006, paper OFD3. 
[5] R. Freund, L. Molle, C. Caspar, J. Schwartz, S. Webb, and S. Barnes, "Mixed bitrate and modulation format upgrades of non-slope matched submarine links," in Proc. of 32nd European Conference on Optical Communication (ECOC 2006), Cannes, France, Sept. 2006, paper Th1.6.5.

[6] B. Slater, S. Boscolo, T. Broderick, S. K. Turitsyn, R. Freund, L. Molle, C. Caspar, J. Schwartz, and S. Barnes, "Performance analysis of $20 \mathrm{~Gb} / \mathrm{s}$ RZ-DPSK non-slope matched transoceanic submarine links," Opt. Express, vol. 15 pp. 10999-11007, 2007.

[7] L. Molle, C. Caspar, R. Freund, S. Desbruskais, R. Oberland, and J. Schwartz, "Upgrades of non-slope matched submarine transmission systems using differential phase shift keying," in Tech. Dig. of Optical Fiber Communication Conference (OFC 2007), Anaheim, CA, Mar. 2007, paper OWM2.

[8] L. Becouarn, G. Vareille, P. Pecci, and J. F. Marcerou, "3 Tbit/s transmission (301 DPSK channels at $10.709 \mathrm{~Gb} / \mathrm{s}$ ) over $10270 \mathrm{~km}$ with a record efficiency of $0.65 \mathrm{bit} / \mathrm{s} / \mathrm{Hz}$," in Proc. of 29th European Conference on Optical Communication (ECOC 2003), Rimini, Italy, Sept. 2003, postdeadline paper Th4.3.2.

[9] A. H. Gnauck, G. Raybon, S. Chandrasekhar, J. Leuthold, C. Doerr, L. Stulz, A. Agarwal, S. Banerjee, D. Grosz, S. Hunsche, A. Kung, A. Marhelyuk, D. Maywar, M. Movassaghi, X. Liu, C. Xu, X. Wei, and D. M. Gill, "2.5 Tb/s (64 x $42.7 \mathrm{~Gb} / \mathrm{s}$ ) transmission over 40 x $100 \mathrm{~km}$ NZDSF using RZ-DPSK format and all-Raman-amplified spans," in Tech. Dig. of Optical Fiber Communication Conference (OFC 2002), Anaheim, CA, Mar. 2002, postdeadline paper FC2.

[10] A. N. Pilipetskii, "Propagation effects at high bit rates," in Tech. Dig. of Optical Fiber Communication Conference (OFC 2006), Anaheim, CA, Mar. 2006, paper OWJ7.

[11] N. S. Bergano, F. W. Kerfoot, and C. R. Davidson, "Margin measurements in optical amplifier system," IEEE Photon. Technol. Lett., vol. 5, pp. 304-306, 1993.

[12] C. Xu, X. Liu, and X. Wei, "Differential phase-shift keying for high spectral efficiency optical transmissions," IEEE J. Sel. Top. Quantum Electron., vol. 10, pp. 281-293, 2004.

[13] B. Slater, S. Boscolo, V. K. Mezentsev, and S. K. Turitsyn, "Comparative analysis of BER estimation methods in numerical simulation of 40-Gb/s RZ-DPSK transmission with in-line SOAs,” IEEE Photon. Technol. Lett., vol. 19, pp. 607-609, 2007.

[14] R. J. Essiambre, B. Mikkelsen and G. Raybon "Intra-channel cross-phase modulation and four-wave mixing in highspeed TDM systems" Electron. Lett., vol. 35, pp. 1576-1578, 1999.

[15] T. Broderick, B. Slater, S. Boscolo, and S. K. Turitsyn, "Statistical analysis of RZ-DPSK fiber communication channels," in Proc. of XVI International Workshop on Optical Waveguide Theory and Numerical Modelling (OWTNM 2007), Copenhagen, Denmark, Apr. 2007, paper PO-01.04.

[16] A. H. Gnauck and P. J. Winzer, “Optical phase shift keyed transmission,” J. Lightwave Technol., vol. 23, pp. 155169, 2006.

[17] F. Forgieri, R. W. Tkach, and A. R. Chraplyvy, "Fiber nonlinearities and their impact on transmission systems," in Optical Fiber Communications vol. 3 A, I. P. Kaminow and T. L. Koch, Eds., New York: Academic, 1997, ch. 8.

[18] T. Broderick "Estimation of bit error rate for pseudo-linear differential phase shift keyed transmission links," Optics Commun., 2008, Submitted. 